\title{
Abnormal origin of the left coronary artery corrected with modified Lecompte maneuver
}

\section{Origen anómalo de la coronaria izquierda corregido con maniobra modificada de Lecompte}

\author{
Adrián Bolívar-Mejía ${ }^{* *}$, Pedro Abad², Carlos Martínez ${ }^{3}$, Juan C. Rendón ${ }^{4}$, Jorge Zapata ${ }^{4}$ and \\ Clara Saldarriaga-Giraldo 5
}

${ }^{1}$ Cardiology Department, Clínica Cardio VID. Cardiology Postgraduate Program, Universidad Pontifica Bolivariana; ${ }^{2}$ Cardio-imaging consultant and diagnostic aid radiologist, SURA; ${ }^{3}$ Cardiology Department, Clínica Cardio VID; ${ }^{4}$ Department of Cardiovascular Surgery, Clínica Cardio VID, Cardiology Postgraduate Program Coordination, Universidad Pontificia Bolivariana; ${ }^{5}$ Cardiology and Heart Failure Department, Clínica Cardio VID. Medellin Colombia

Normal cardiac irrigation is driven by the origin the right and left coronary arteries at the level of the right and left coronary sinuses; however, modifications of the described anatomy can occur, some of which correspond to harmless variants, while others may be associated with high risk of morbidity and mortality ${ }^{1,2}$.

\section{Clinical case}

Fifty-four-year-old woman with a history of hypercholesterolemia controlled with rosuvastatin $(10 \mathrm{mg} /$ day $)$. She referred 4 months of chest pain, stabbing in nature, which appeared indistinctly at rest or exercise. Occasionally, it was associated with dyspnea, radiating to interscapular region. Physical examination and electrocardiogram were normal. Exercise stress echocardiogram was submaximal (75\%), inconclusive for ischemia. Left ventricular function was normal.

Risk re-stratification with coronary calcium score was indicated, which reported an Agatston score of 0 , with possible right coronary artery anomalous origin being additionally described. Coronary computed tomography angiography reported a right coronary artery of normal origin and course, and left main trunk anomalous origin,
$5 \mathrm{~mm}$ cephalad to the sinotubular junction above the right coronary sinus, which ran from the aorta to the pulmonary artery (Fig. 1). Coronary angiography ruled out atherosclerotic disease.

The patient underwent left coronary artery decompression with modified Lecompte maneuver (Fig. 2), and had an adequate postoperative evolution. On follow-up visit 5 months after surgical intervention, she was asymptomatic, with New York Heart Association functional class I/IV.

\section{Discussion}

Coronary arteries anomalous origin corresponds to congenital alterations present in less than $1 \%$ of the population, which can be asymptomatic or generate episodes of angina, dyspnea, syncope or sudden cardiac death ${ }^{1,2}$.

The most common anomaly is anterior descending and circumflex arteries origin from different ostia, a finding that usually is not associated with hemodynamic repercussions ${ }^{1,2}$. Other less common alterations are contralateral origin of the coronary arteries ostium or presence of a single coronary artery ${ }^{1.3}$. Once the vessel
Available online: $20-10-2021$ Arch Cardiol Mex (Eng). 2021;91(3):351-353 www.archivoscardiologia.com license (http://creativecommons.org/licenses/by-nc-nd/4.0/). 


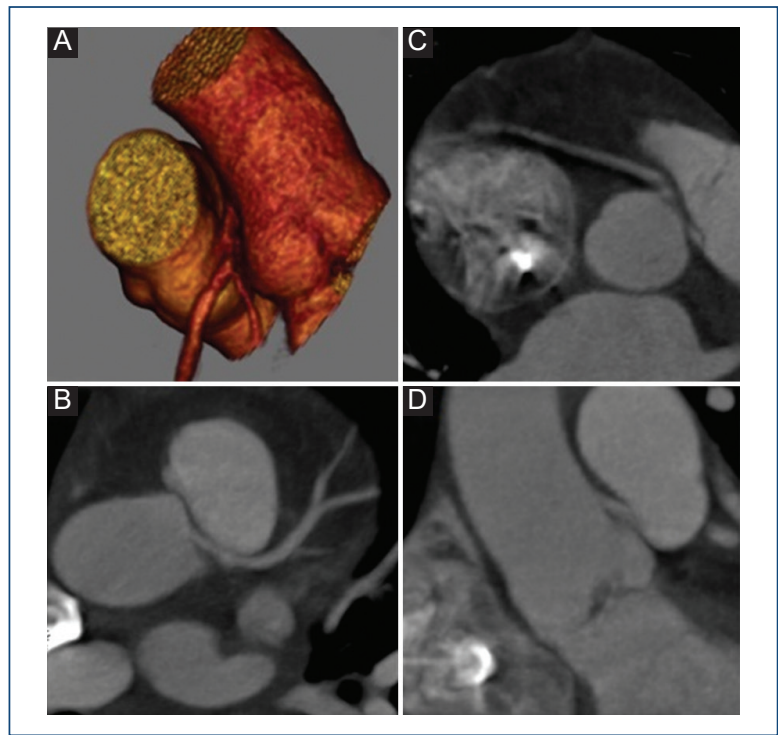

Figure 1. Coronary computed tomography angiography. A: three-dimensional reconstruction showing left coronary trunk interarterial course with no evidence of intramural path. B: transverse view showing evidence of right coronary artery normal origin and course. C and D: transverse (C) and coronal (D) view of left coronary artery anomalous origin and interarterial course.

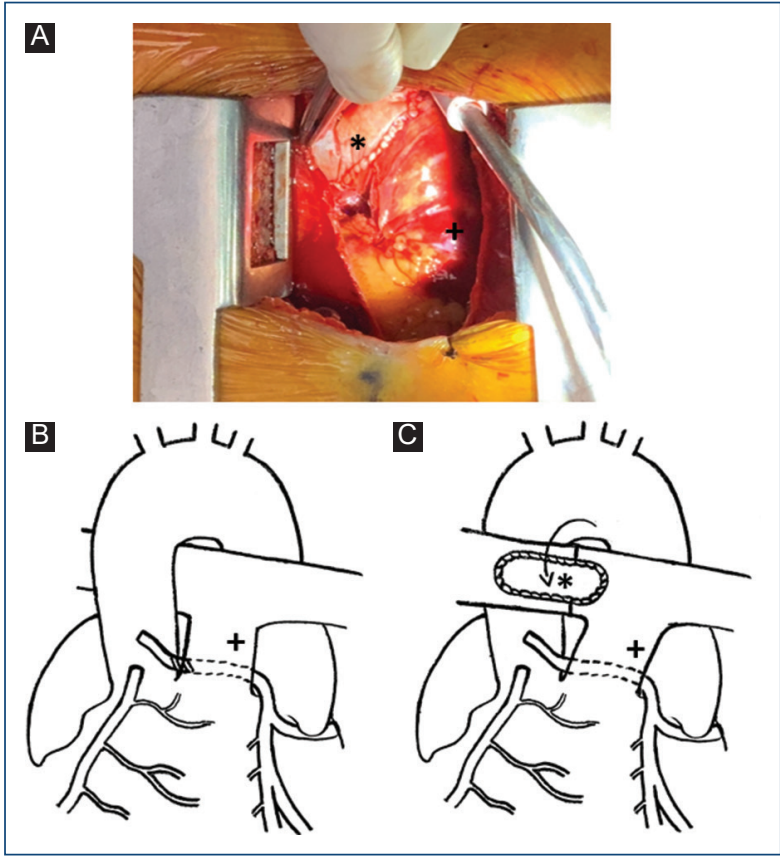

Figure 2. A: photograph of the intraoperative result of our patient after the performance of modified Lecompte maneuver. B and C: depiction of pulmonary artery trunk translocation by modified Lecompte maneuver. +: pulmonary artery trunk. *: pericardial patch on the pulmonary artery right branch. arises from the contralateral coronary sinus it can take different courses: anterior to the pulmonary artery trunk, retroaortic, subpulmonary intramyocardial through the septum, retrocardiac or interarterial. ${ }^{1}$. The risk of sudden death is related to coronary anomaly anatomical characteristics. Thus, a course between the aorta and the pulmonary artery trunk, as in the presented case, often determines a higher risk of sudden death due to compression between both these vessels. Other abnormalities associated with sudden death include left coronary artery anomalous origin from the pulmonary artery, left coronary artery main trunk atresia and presence of coronary fistulae ${ }^{1,2}$.

The diagnostic process should be based on patient clinical manifestations, considering that symptoms can be atypical, and when it occurs in usually young patients without cardiovascular risk factors, this can lead to underestimate the probability of ischemic heart disease ${ }^{4}$.

Diagnostic tools include coronary computed tomography angiography, cardiac magnetic resonance and coronary arteriography, which can be accompanied by intravascular ultrasound and optical coherence tomography, which are tools that allow evaluating the vessel lumen with higher level of detail and that provide relevant information to define the need for surgical intervention and the surgical technique to be employed ${ }^{3-5}$.

Surgical management is indicated whenever symptoms or findings consistent with ischemia associated with the coronary anomaly are documented in complementary studies (recommendation IB), and it is reasonable (recommendation Ila) in patients with left coronary artery origin in the right coronary sinus even in the absence of symptoms, a scenario in which the previously-described anatomical aspects associated with high risk should be taken into account ${ }^{5}$.

Different surgical strategies have been described, which depend on the coronary anomaly origin anatomical characteristics and the vessel course after its emergence from the ostium ${ }^{6}$. Modified Lecompte maneuver is a procedure for the management of coronary anomalous origin whose course does not imply an intramural path ${ }^{6}$. It consists of an anterior translocation of the pulmonary artery trunk that allows the interarterial space between the vessel and the aorta to be widened, and thereby avoid compression of the coronary artery with interarterial course and omit the need for compromised vessel re-implantation ${ }^{6}$. For this reason, coronary anatomy of our patient made her a suitable candidate for this intervention, which was uneventfully performed, 
with an adequate postoperative evolution and hospital discharge 3 days after surgery being achieved, and the patient being found free of cardiovascular symptoms after 5 months of postoperative follow-up.

\section{Conclusion}

The presented case reflects the importance of chest pain individualized approach, which should not be biased by the presence or absence of classic cardiovascular risk factors or framed within traditionally used risk scores, given that ischemic heart disease is not limited to atherosclerotic disease and sometimes can be explained by the presence of coronary circulation congenital anomalies.

\section{Conflicts of interests}

None.

\section{Funding}

This investigation has not received any specific grant from agencies of the public, commercial or non-profit sectors.

\section{Ethical disclosures}

Protection of human and animal subjects. The authors declare that no experiments have been performed on humans or animals for this investigation.

Confidentiality of data. The authors declare that they have followed the protocols of their work center on the publication of patient data.

Right to privacy and informed consent. The authors declare that no patient data appear in this article.

\section{References}

1. Harky A, Noshirwani A, Karadakhy O, Ang J. Comprehensive literature review of anomalies of the coronary arteries. J Card Surg. 2019;34:1328-43.

2. Kastellanos S, Aznaouridis K, Vlachopoulos C, Tsiamis E, Oikonomou E, Tousoulis D. Overview of coronary artery variants, aberrations and anomalies. World J Cardiol. 2018;10:127-40.

3. Martín M, Avanzas P, Corros C, Fernández-Cimadevilla QC, León D, Barreiro $\mathrm{M}$, et al. Arteria coronaria única con origen en seno de Valsalva derecho. Utilidad de la angiotomografía coronaria con tomografía multicorte. Cardiol Mex. 2011;81:327-9.

4. Gayás Ordás J, Linares Vicente JA, Angulo Hervias E, Revilla Martí P Ruiz Arroyo JR. ¿Dónde está el tronco coronario izquierdo? Cardiol Mex. 2018;88:514-7.

5. Stout KK, Daniels CJ, Aboulhosn JA, Bozkurt B, Broberg CS, Colman JM, et al. 2018 AHA/ACC Guideline for the Management of Adults With Congenital Heart Disease: A Report of the American College of Cardiology/American Heart Association Task Force on Clinical Practice Guidelines. J Am Coll Cardiol. 2019;73:e81-e192.

6. Mainwaring RD, Reddy VM, Reinhartz O, Petrossian E, Punn R, Hanley FL. Surgical repair of anomalous aortic origin of a coronary artery. Eur J Cardiothorac Surg. 2014;46:20-6. 